\title{
A Study on Iterative Algorithm for Stochastic Distribution Free Inventory Models
}

\author{
Jennifer Lin \\ Department of Transportation Logistics \& Marketing Management, Toko University, Chiayi County 61363, Taiwan \\ Correspondence should be addressed to Jennifer Lin; jennifer1592001@yahoo.com.tw
}

Received 27 September 2012; Accepted 13 February 2013

Academic Editor: Somyot Plubtieng

Copyright ( 2013 Jennifer Lin. This is an open access article distributed under the Creative Commons Attribution License, which permits unrestricted use, distribution, and reproduction in any medium, provided the original work is properly cited.

\begin{abstract}
We studied the iterative algorithm in Tung et al. (2010) to find out that their assertion is questionable. We derived two new relations between safe factor and order quantity so that we can execute the iterative algorithm proposed by Wu and Ouyang (2001). We have proved that three generated sequences indeed converge to provide a theoretical validation for their iterative procedure.
\end{abstract}

\section{Introduction}

Paknejad et al. [1] developed inventory models where lead time and defective rate are constant. Wu and Ouyang [2] generalized their results to include crashable lead time, and defective items are random variables that follow a probabilistic distribution with known mean and derivation. For the distribution free inventory model, Wu and Ouyang [2] used the minimax approach of Moon and Gallego [3] to study the minimum problem for an upper bound for the expected average cost. Wu and Ouyang [2] mentioned that the optimal order quantity and safety factor can be derived by iterative algorithm. Tung et al. [4] developed an analytical approach to prove that the optimal solutions for the order quantity and safety factor exist and are unique. Moreover, they claimed that iterative algorithm cannot be operated by the formulas in $\mathrm{Wu}$ and Ouyang [2].

Tung et al. [4] offered an analytical proof to prove the existence and uniqueness of the optimal solution for the inventory model of Wu and Ouyang [2]. During their derivations, they found two upper bounds and one lower bound, and then they used numerical examination to compare these two upper bounds to decide the minimum upper bound. They only studied the first derivative system such that they only considered the interior minimum. Moreover, they examined the iterative algorithm in $\mathrm{Wu}$ and Ouyang [2] to claim that the formulas in $\mathrm{Wu}$ and Ouyang [2] cannot be used to locate the optimal order quantity and safety factor. In this paper, we will show that the formulas in $\mathrm{Wu}$ and
Ouyang [2] after two modifications are workable for the iterative algorithm.

\section{Review of Previous Results}

To be compatible with the results of Wu and Ouyang [2] and Tung et al. [4], we use the same notation and assumptions. We study the paper of Tung et al. [4]. They considered the stochastic inventory model of Wu and Ouyang [2] with crashable lead time, defective items, and minimax approach for distribution free demand with the following objective function:

$$
\begin{aligned}
E A C^{u}(Q, k, L)= & \frac{A D}{Q(1-E(p))} \\
+ & \frac{h}{2}\left\{Q(1-E(p))+Q \frac{E\left(p^{2}\right)-E^{2}(p)}{1-E(p)}\right. \\
& \left.\quad+\frac{E(p(1-p))}{1-E(p)}\right\} \\
+ & h\left\{k \sigma \sqrt{L}+\frac{1-\beta}{2} \sigma \sqrt{L}\left(\sqrt{1+k^{2}}-k\right)\right\} \\
& +\frac{D\left(\pi+\pi_{0}(1-\beta)\right)}{2 Q(1-E(p))} \sigma \sqrt{L}\left(\sqrt{1+k^{2}}-k\right)
\end{aligned}
$$




$$
\begin{aligned}
& +(Q-1) h^{\prime} \frac{E(p(1-p))}{1-E(p)} \\
& +\frac{D v}{1-E(p)}+\frac{D}{Q(1-E(p))} \\
& \times\left(c_{i}\left(L_{i-1}-L\right)+\sum_{j=1}^{i-1} c_{j}\left(b_{j}-a_{j}\right)\right),
\end{aligned}
$$

for $L \in\left[L_{i}, L_{i-1}\right]$, where $\operatorname{EAC}^{\mathcal{u}}(Q, k, L)$ is a least upper bound of $\operatorname{EAC}(Q, k, L)$. Wu and Ouyang [2] derived that $E A C^{u}(Q, k, L)$ is a concave function of $L$ with $L \in\left[L_{i}, L_{i-1}\right]$; so, the minimum must occur at boundary point $L_{i}$ or $L_{i-1}$. To simplify the expression, we will use $L$ instead of $L_{i}$ or $L_{i-1}$ as Tung et al. [4]. For $\operatorname{EAC}^{u}(Q, k, L)$, Wu and Ouyang [2] computed the first partial derivatives with respect to $Q$ and $k$, separately.

Using $(\partial / \partial Q) E A C^{u}(Q, k, L)=0$ and $(\partial / \partial k) E A C^{u}(Q$, $k, L)=0$, Wu and Ouyang [2] derived that

$$
\begin{array}{r}
Q=\left[\frac { 2 D } { h \delta } \left\{A+c_{i}\left(L_{i-1}-L\right)+\sum_{j=1}^{i-1} c_{j}\left(b_{j}-a_{j}\right)\right.\right. \\
\left.\left.+\frac{\pi+\pi_{0}(1-\beta)}{2} \sigma \sqrt{L}\left(\sqrt{1+k^{2}}-k\right)\right\}\right]^{1 / 2},
\end{array}
$$

where $\delta=1-2 E(p)+E\left(p^{2}\right)+2\left(h^{\prime} / h\right) E(p(1-p))$, and

$$
\frac{2 \sqrt{1+k^{2}}}{\sqrt{1+k^{2}}-k}=1-\beta+\frac{D\left(\pi+\pi_{0}(1-\beta)\right)}{h Q(1-E(p))} \text {. }
$$

Wu and Ouyang [2] claimed that the optimal solution can be obtained by the iterative method. In Tung et al. [4], they assumed that $k_{0}=0$ to plug it into (2) to derive $Q_{1}=315.62$, and then they plugged it into (3) to derive a relation for $k_{1}$ as

$$
\frac{\sqrt{1+k_{1}^{2}}}{\sqrt{1+k_{1}^{2}}-k_{1}}=11.9811
$$

Tung et al. [4] mentioned that, in (4), they only derived the value for $\sqrt{1+k_{1}^{2}} /\left(\sqrt{1+k_{1}^{2}}-k_{1}\right)$ such that they can not use (3) to execute the iterative process. In this paper, we will show that (3) can be improved to operate the iterative process.

\section{Our Revision for Tung et al. [4]}

When running the iterative process, after we derive $Q_{n}$, we then plug it into (3) to find a relation of $k_{n}$ as

$$
\frac{\sqrt{1+k_{n}^{2}}}{\sqrt{1+k_{n}^{2}}-k_{n}}=\frac{1}{2}\left(1-\beta+\frac{D\left(\pi+\pi_{0}(1-\beta)\right)}{h Q_{n}(1-E(p))}\right) .
$$

To abstractly handle this problem, we assume that

$$
\frac{1}{2}\left(1-\beta+\frac{D\left(\pi+\pi_{0}(1-\beta)\right)}{h Q_{n}(1-E(p))}\right)=d_{n} .
$$

Under the assumption of Tung et al. [4], it yields that

$$
Q<\sqrt{\alpha_{1}+\alpha_{2}}<\frac{\alpha_{4}}{(1+\beta) \alpha_{3}}
$$

with $\alpha_{3}=h(1-E(p))$, and $\alpha_{4}=D\left(\pi+\pi_{0}(1-\beta)\right)$ to imply that

$$
d_{n}=\frac{1}{2}\left(1-\beta+\frac{\alpha_{4}}{\alpha_{3} Q_{n}}\right)>\frac{1}{2}(1-\beta+1+\beta)=1 .
$$

From (5) and (6), and with $d_{n}>1$, we obtain that

$$
\sqrt{1+k_{n}^{2}}\left(d_{n}-1\right)=d_{n} k_{n}
$$

and then squaring both sides, we find that

$$
k_{n}=\frac{d_{n}-1}{\sqrt{2 d_{n}-1}}
$$

to show that there is a unique $k_{n}$ that can be derived by (3). The assertion of Tung et al. [4] that (3) can not be used to execute the iterative process can be improved.

\section{The Proof for the Convergence of the Proposed Three Iterated Sequences}

In this section, we will prove that the two iterative sequences proposed for (2) and (10) indeed converge.

We combine (6) and (10) to derive that

$$
k_{n}=\frac{D\left(\pi+\pi_{0}(1-\beta)\right) / h Q_{n}(1-E(p))-(1+\beta)}{2\left(D\left(\pi+\pi_{0}(1-\beta)\right) / h Q_{n}(1-E(p))-\beta\right)^{1 / 2}} .
$$

For later purpose, we rewrite the iterative process based on (2) as follows:

$$
Q_{n+1}=\left(B_{1}+B_{2}\left(\sqrt{1+k_{n}^{2}}-k_{n}\right)\right)^{1 / 2}
$$

where $B_{1}=\left[(2 D / h \delta)\left\{A+c_{i}\left(L_{i-1}-L\right)+\sum_{j=1}^{i-1} c_{j}\left(b_{j}-a_{j}\right)\right\}\right]$ and $B_{2}=\left[(2 D / h \delta)\left\{\left(\left(\pi+\pi_{0}(1-\beta)\right) / 2\right) \sigma \sqrt{L}\right\}\right]$ with $B_{1}>0$ and $B_{2}>0$.

Based on (12), we derive that

$$
\begin{aligned}
Q_{n+2}^{2}-Q_{n+1}^{2} & =B_{2}\left[\left(k_{n}-k_{n+1}\right)+\sqrt{1+k_{n+1}^{2}}-\sqrt{1+k_{n}^{2}}\right] \\
& =B_{2}\left[\left(k_{n}-k_{n+1}\right)+\frac{k_{n+1}^{2}-k_{n}^{2}}{\sqrt{1+k_{n+1}^{2}}+\sqrt{1+k_{n}^{2}}}\right] \\
& =B_{2}\left(k_{n+1}-k_{n}\right)\left[\frac{k_{n+1}+k_{n}}{\sqrt{1+k_{n+1}^{2}}+\sqrt{1+k_{n}^{2}}}-1\right] .
\end{aligned}
$$


We know that $\sqrt{1+x^{2}}>x$ such that $\left(k_{n+1}+k_{n}\right) /\left(\sqrt{1+k_{n+1}^{2}}+\right.$ $\left.\sqrt{1+k_{n}^{2}}\right)-1<0$. Hence, we obtain the following lemma.

Lemma 1. If $k_{n+1}>k_{n}$, then $Q_{n+2}<Q_{n+1}$.

One recalls (10); then one evaluates that

$$
\begin{aligned}
k_{n+1}^{2}-k_{n}^{2} & =\frac{\left(d_{n+1}-1\right)^{2}}{2 d_{n+1}-1}-\frac{\left(d_{n}-1\right)^{2}}{2 d_{n}-1} \\
& =\frac{d_{n+1}^{2}-2 d_{n+1}+1}{2 d_{n+1}-1}-\frac{d_{n}^{2}-2 d_{n}+1}{2 d_{n}-1} \\
& =\frac{d_{n+1}^{2}}{2 d_{n+1}-1}-\frac{d_{n}^{2}}{2 d_{n}-1} .
\end{aligned}
$$

We need the next lemma for our future proof.

Lemma 2. If $d_{n+1}>d_{n}>1$, then $k_{n+1}>k_{n}$.

After cross-multiplication of (14), one finds that $k_{n+1}^{2}>k_{n}^{2}$ if and only if

$$
d_{n}\left(2 d_{n+1}^{2}+d_{n}\right)>d_{n+1}\left(d_{n+1}+2 d_{n}^{2}\right)
$$

that is,

$$
\frac{2 d_{n+1}^{2}+d_{n}}{d_{n+1}}>\frac{2 d_{n}^{2}+d_{n+1}}{d_{n}} .
$$

One rewrites (16) as follows:

$$
2 d_{n+1}+\frac{d_{n}}{d_{n+1}}>2 d_{n}+\frac{d_{n+1}}{d_{n}}
$$

that is equivalent to

$$
2\left(d_{n+1}-d_{n}\right)>\frac{d_{n+1}}{d_{n}}-\frac{d_{n}}{d_{n+1}}=\frac{d_{n+1}^{2}-d_{n}^{2}}{d_{n} d_{n+1}} .
$$

One can cancel out the common factor $d_{n+1}-d_{n}>0$ from the previous inequality and still preserve the same direction of the inequality sign.

Consequently, one tries to show that

$$
2 d_{n} d_{n+1}>d_{n}+d_{n+1} \text {. }
$$

One knows that $2 d_{n} d_{n+1}-d_{n}-d_{n+1}=d_{n}\left(d_{n+1}-1\right)+d_{n+1}\left(d_{n}-\right.$ 1) $>0$, owing to the condition of $d_{n+1}>d_{n}>1$.

From the iterative procedure, $k_{0}=0$, which is plugged it into (2) to derive $Q_{1}>0$, and then we plug $Q_{1}$ into (11) to obtain that $k_{1}>0$.

Owing to $k_{1}>k_{0}$, we apply Lemma 1 to obtain $Q_{2}<Q_{1}$.

We may simplify (6) as follows:

$$
d_{n}=B_{3}+\frac{B_{4}}{Q_{n}}
$$

where $B_{3}=(1-\beta) / 2$ and $B_{4}=\left(D\left(\pi+\pi_{0}(1-\beta)\right) / 2 h(1-E(p))\right)$ with $B_{3} \geq 0$ and $B_{4}>0$.
TABLE 1: For $\beta=0.5$ and $L_{3}=3$, the convergence of proposed three sequences.

\begin{tabular}{cccc}
\hline$i$ & $k_{i}$ & $Q_{i}$ & $d_{i}$ \\
\hline 0 & 0 & & \\
1 & 1.925382 & 271.444019 & 8.884377 \\
2 & 2.433960 & 179.201796 & 13.328831 \\
3 & 2.490503 & 171.872873 & 13.886532 \\
4 & 2.495817 & 171.206597 & 13.939601 \\
5 & 2.496308 & 171.145237 & 13.944509 \\
6 & 2.496353 & 171.139579 & 13.944962 \\
7 & 2.496357 & 171.139060 & 13.945003 \\
8 & 2.496357 & 171.139014 & 13.945007 \\
9 & 2.496357 & 171.139014 & 13.945007 \\
\hline
\end{tabular}

Using $Q_{2}<Q_{1}$, by (20), and we have $d_{2}>d_{1}$, applying Lemma 2, we know that $k_{2}>k_{1}$.

Repeating the previous argument, we derived that $\left(k_{n}\right)$ is an increasing sequence and $\left(Q_{n}\right)$ is a decreasing sequence and bounded below by zero such that $\left(Q_{n}\right)$ must converge, and then sequence $\left(d_{n}\right)$ converges. Finally, the sequence $\left(k_{n}\right)$ converges too.

\section{Numerical Example to Support Our Proof}

We will use the same numerical examples in $\mathrm{Wu}$ and Ouyang [2] and Tung et al. [4] to illustrate our findings in Section 5. For the precious space of this journal, please refer to $\mathrm{Wu}$ and Ouyang [2] for the detailed data, and we refer to the findings of Tung et al. [4] to consider the representative case of $\beta=0.5$ and $L_{3}=3$ to demonstrate the convergence of the proposed two sequences $\left(k_{i}\right)_{i \geq 0}$ and $\left(Q_{i}\right)_{i \geq 1}$ with our auxiliary sequence $\left(d_{i}\right)_{i \geq 1}$. We list the numerical results in Table 1.

If we observe Table 1 , then the sequence $\left(k_{i}\right)_{i \geq 0}$ increases to its least upper bound and the sequence $\left(Q_{i}\right)_{i \geq 1}$ decreases to its greatest lower bound as described by our proposed analysis. Our finding is consistent with Tung et al. [4] in which they mentioned that for $\beta=0.5$ and $L_{3}=3, Q^{*}=$ 171.14

\section{References}

[1] M. J. Paknejad, F. Nasri, and J. F. Affisco, "Defective units in a continuous review (s, Q) system," International Journal of Production Research, vol. 33, pp. 2767-2777, 1995.

[2] K. S. Wu and L. Y. Ouyang, "(Q, r, L) Inventory model with defective items," Computers and Industrial Engineering, vol. 39, no. 1-2, pp. 173-185, 2001.

[3] I. Moon and G. Gallego, "Distribution free procedures for some inventory models," Journal of Operational Research Society, vol. 45, no. 6, pp. 651-658, 1994.

[4] C.-T. Tung, Y.-W. Wou, S.-W. Lin, and P. Deng, "Technical note on $(Q, r, L)$ inventory model with defective items," Abstract and Applied Analysis, vol. 2010, Article ID 878645, 8 pages, 2010. 


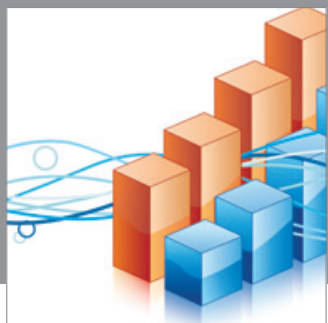

Advances in

Operations Research

mansans

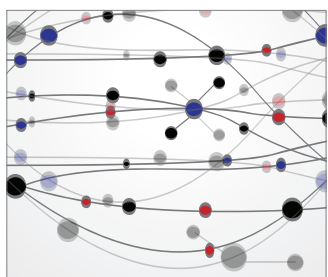

The Scientific World Journal
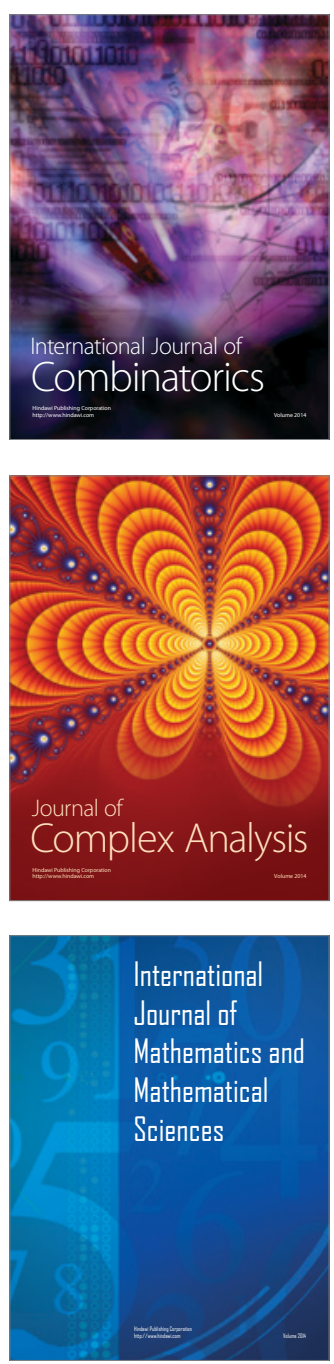
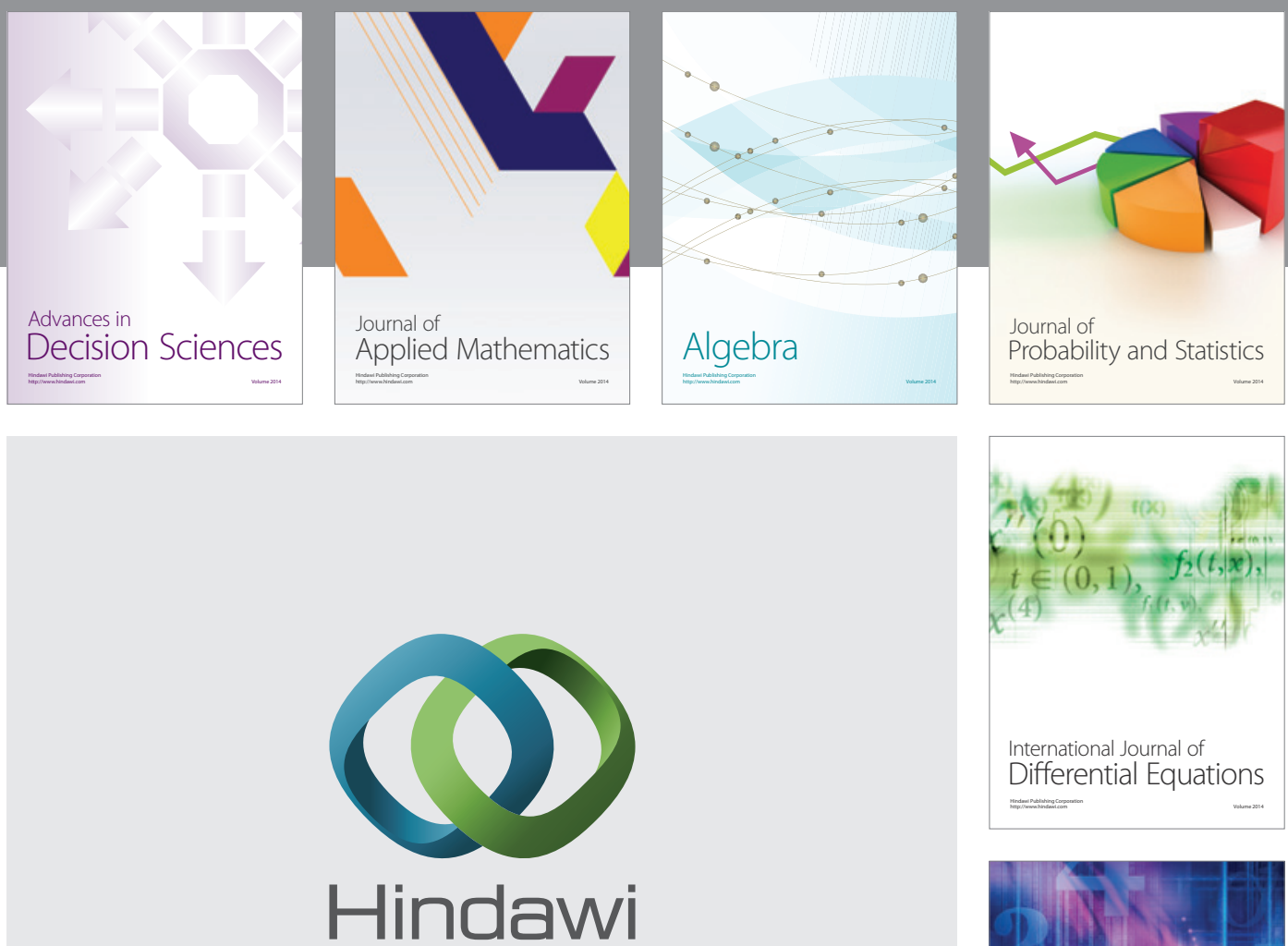

Submit your manuscripts at http://www.hindawi.com
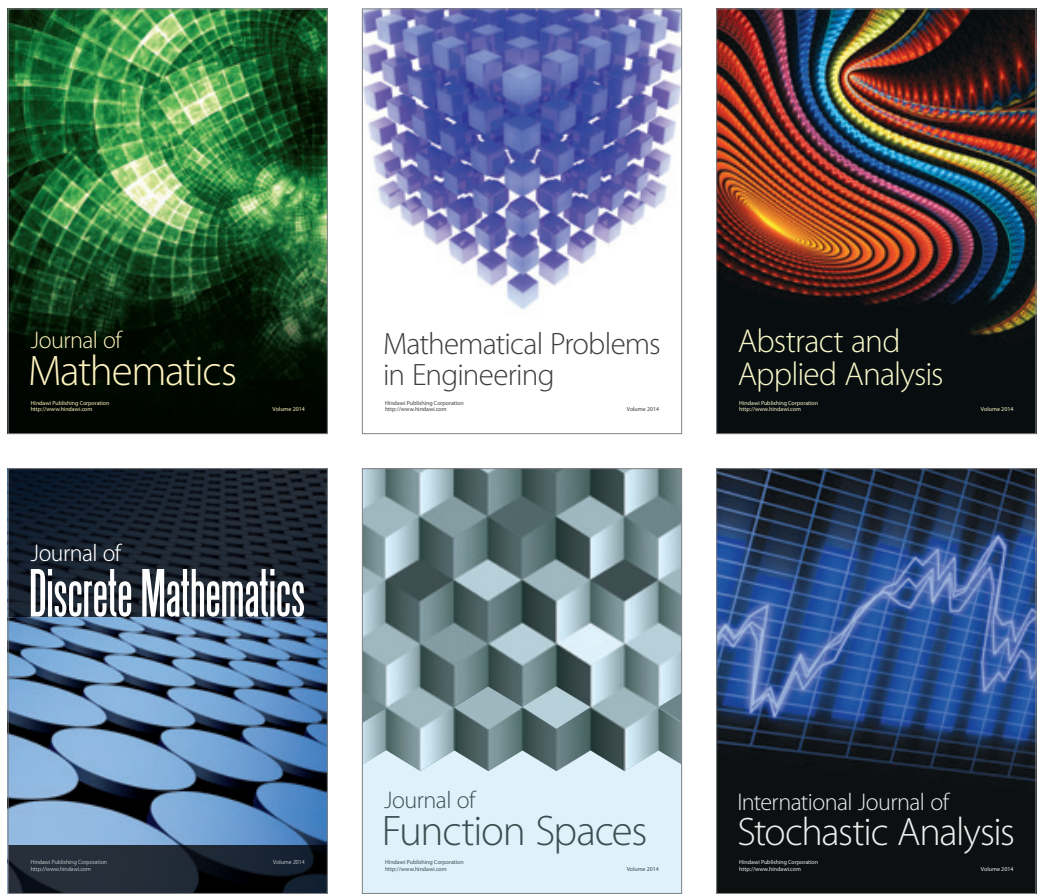

Journal of

Function Spaces

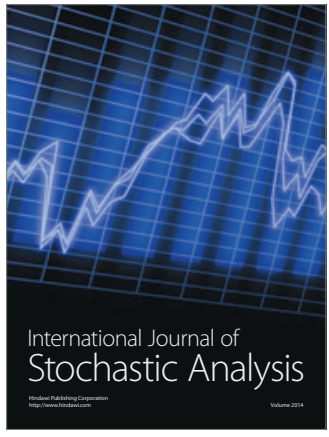

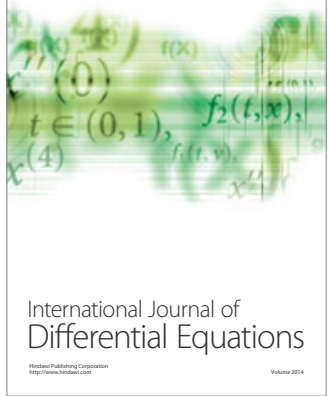
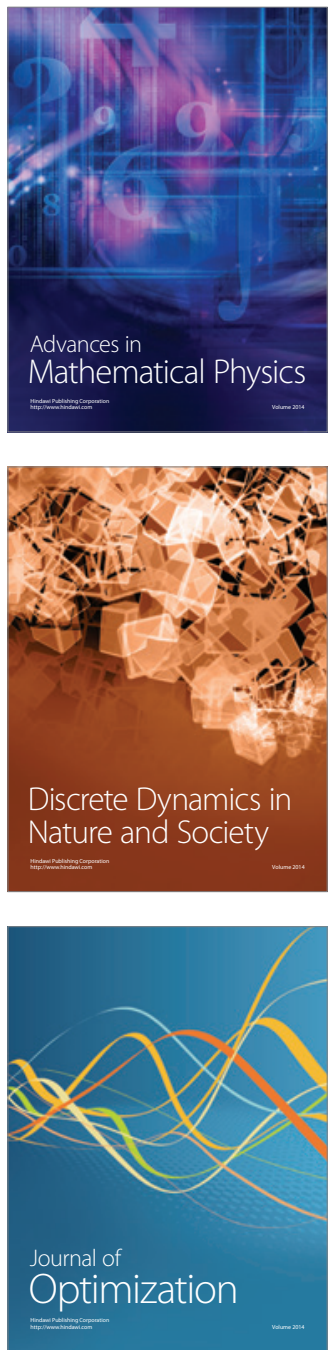